\section{Design and Methodology of the Strategies to Reduce Transmission of Antimicrobial Resistant Bacteria in Intensive Care Units (STAR-ICU) Trial}

TO THE EDITOR-In the October 2006 issue of this journal, Dr. Barry Farr presented a critique ${ }^{1}$ of several published studies and our recently completed, but as yet unreported, multicenter, cluster randomized trial, the Strategies to Reduce Transmission of Antimicrobial Resistant Bacteria in Intensive Care Units (STAR-ICU) trial, a study sponsored by the National Institutes of Allergy and Infectious Diseases (NIAID) and conducted through the Bacteriology and Mycology Study Group (BAMSG) (ClinicalTrials.gov Identifier: NCT00342745). ${ }^{2}$ The results of the trial are currently being analyzed and will be reported in early 2007 . This letter outlines features of the study that enhance its internal and external validity and addresses Dr. Farr's principal criticisms. We encourage readers to judge the study on the basis of a complete discussion of its design and methodology and a full report of its results.

The STAR-ICU trial was not intended to test the specific recommendations of the 2003 Society for Healthcare Epidemiology in America (SHEA) guideline for control of methicillin-resistant Staphylococcus aureus (MRSA) or vancomycin-resistant Enterococcus (VRE). ${ }^{3}$ We share Dr. Farr's belief that substantial literature has established that active surveillance is necessary to identify patients who are asymptomatically colonized with MRSA or VRE and that contact precautions can interrupt direct or indirect transmission of MRSA or VRE from colonized patients. The trial was not devised to reassess these issues.

Rather, the trial was designed to rigorously address an important and practical clinical question: "Does an intensive infection control strategy (a strategy that uses surveillance cultures on admission and at weekly intervals to identify patients who are colonized with MRSA or VRE, contact precautions for care of patients who are colonized or infected with MRSA or VRE, and a program to promote hand hygiene and standard precautions) reduce the endemic incidence of MRSA and VRE colonization or infection in adult ICUs more than a standard infection control strategy does (a strategy that includes a program to promote hand hygiene and standard precautions, as well as existing unit-defined isolation precautions for care of patients who are colonized or infected with MRSA or VRE)?" Only limited data in the peer-reviewed literature directly address this question, and no data are available from a randomized, controlled trial. We believe many people are keenly interested in the answer to this question.
Two of the strengths of the trial, which were not mentioned by Dr. Farr, ${ }^{1}$ are that it is multicentered and cluster-based (ICU-based). The enrollment of multiple sites distributed across the United States enhances the generalizability of the results and reduces the likelihood that regional differences in the prevalence of MRSA or VRE colonization, especially colonization with community-acquired MRSA strains, will confound its findings. A cluster-based design is important because MRSA and VRE are transmissible; consequently, an individual patient's risk of becoming colonized with MRSA or VRE is dependent on the prevalence of colonization among other patients in the same ICU. This nonindependence of colonization events has been ignored in the design and analysis of previous studies and increases the likelihood of a falsepositive conclusion about the effectiveness of using active surveillance and contact precautions.

Dr. Farr ${ }^{1}$ contends that the study is likely to be under-powered because it has a "short" intervention period. Using data from Project Intensive Care Antimicrobial Resistance Epidemiology (ICARE), ${ }^{4}$ we characterized the 2 sources of variability that are likely to affect the power of the study: the variability that occurs temporally within a unit and the unit-to-unit variability. On the basis of the ICARE data, we determined that an intervention period of 6 months provided sufficient patientdays such that within-unit variability would contribute only approximately $10 \%-15 \%$ of the total variance in the final parameter estimates; the remainder of the variance would be the result of unit-to-unit variability. Consequently, increasing the duration of the intervention period, as Dr. Farr suggests, ${ }^{1}$ would only minimally enhance the power of the study. Extending the length of the intervention period could enable the trial to detect a delayed effect, to more precisely quantitate an effect that increases progressively over a longer period, and/or to demonstrate the sustainability of the effect. However, extending the intervention period by an additional 6-12 months would have increased the cost of the study substantially.

Dr. Farr states that intervening in "only one unit of a large hospital with a high prevalence is suboptimal." ${ }^{1(\mathrm{p} 1102)} \mathrm{Dr}$. Farr's optimal design, as described in a previous critique, ${ }^{5}$ would be to randomize healthcare facilities or even entire healthcare systems to the intervention and control arms. This was unfeasible. Although there may be benefits to intervening in multiple units within an institution, it is easier to insure reliable implementation of interventions at a microsystem level. Moreover, ICUs are high-risk environments where the reproductive number (ie, the number of sceondary cases from an initial index case) is driven by a high colonization burden and frequent opportunities for transmission.

The study will generate point and interval estimates of the effectiveness of the intensive control strategy compared with the standard control strategy, in reducing the incidence den- 
sities for colonization or infection with either MRSA or VRE, and for colonization or infection with MRSA, and for colonization or infection with VRE. The study is powered to detect a moderate $(40 \%)$ decrease in the composite outcome (MRSA or VRE colonization or infection) associated with the intensive control strategy. A smaller decrease could yield a $P$ value greater than .05 , a negative study result, as noted by Dr. Farr. ${ }^{1}$ However, given that the strategy of using active surveillance and contact precautions strategy involves additional workload and cost, we believed that decision-makers would require at least a moderate reduction to justify wider use of this strategy, especially since many acute care facilities are already committing substantial resources to the prevention of infections in general, not just those caused by MRSA or VRE.

It is premature to judge the contribution of the STAR-ICU trial to the base of evidence regarding the effectiveness of using active surveillance and contact precautions in controlling the spread of MRSA and VRE in healthcare facilities. We encourage readers to evaluate the study critically after its design, methods, results and conclusions have been reported fully.

\section{ACKNOWLEDGMENTS}

The STAR-ICU Trial has been funded in part with Federal Funds from the National Institute of Allergy and Infectious Diseases, National Institutes of Health (contract numbers NO1-AI-15440 and NO1-AI-15441). Additional support has been provided by Merck, Elan Pharmaceuticals, Roche Diagnostics, and Kimberly Clark.

W. Charles Huskins, MD, MSc; Naomi P. O'Grady, MD; Matthew Samore, MD; Dennis Wallace, $\mathrm{PhD}$; Donald A. Goldmann, MD; Peter G. Pappas, MD

From the Mayo Clinic College of Medicine, Rochester, Minnesota (W.C.H.), the National Institutes of Health Clinical Center, Bethesda, Maryland (N.P.O), the University of Utah, Salt Lake City (M.S.), Rho Federal Systems Division, Chapel Hill, North Carolina (D.W.), the Harvard Medical School, Boston, Massachusetts (D.A.G.), and the University of Alabama at Birmingham, Birmingham (P.G.P).

Address reprint requests to $\mathrm{W}$. Charles Huskins, MD, MSc, Mayo Clinic, 200 First Avenue SW, Rochester, MN 55905 (huskins.charles@mayo.edu).

W.C.H. reports having receiving consulting fees from Roche Diagnostics. D.A.G. reports having received research grant support from Clorox and consulting fees from the Cosmetic Toiletry and Fragrance Association, Warner-Lambert, and Merck. P.G.P. reports having received honoraria, consulting fees, and research grant support from Merck. N.P.O., M.S., and D.W. report no conflicts of interest.

Infect Control Hosp Epidemiol 2007; 28:245-246

(C) 2006 by The Society for Healthcare Epidemiology of America. All rights reserved. 0899-823X/2007/2802-0026\$15.00.

\section{REFERENCES}

1. Farr BM. What to think if the results of the National Institutes of Health randomized trial of methicillin-resistant Staphylococcus aureus and vancomycin-resistant Enterococcus control measures are negative (and other advice to young epidemiologists): a review and an au revoir. Infect Control Hosp Epidemiol 2006; 27:1096-1106.

2. ClinicalTrials.gov. Strategies to reduce transmission of antibiotic-resistant bacteria in intensive care units. Available at: http://www.clinicaltrials.gov/ $\mathrm{ct} /$ show/NCT00342745? order = 8. Accessed October 9, 2006.

3. Muto CA, Jernigan JA, Ostrowsky BE, Richet HM, Jarvis WR, Farr BM. SHEA guideline for preventing nosocomial transmission of multidrugresistant strains of Staphylococcus aureus and Enterococcus. Infect Control Hosp Epidemiol 2003; 24:362-386.

4. Fridkin SK, Steward CD, Edwards JR, et al. Surveillance of antimicrobial use and antimicrobial resistance in United States hospitals: project ICARE phase 2. Project Intensive Care Antimicrobial Resistance Epidemiology (ICARE) hospitals. Clin Infect Dis 1999; 29:245-252.

5. Calfee DP, Farr BM. Use of active surveillance cultures to control vancomycin-resistant Enterococcus: reply. Clin Infect Dis 2003; 37:1402-1403.

\section{Reply to Huskins et al.}

TO THE EDITORS-Huskins et al. ${ }^{1}$ say they addressed the "principal criticisms" I made in my article, ${ }^{2}$ but they only discussed inadequate sample size and statistical power and intervention in a single ICU of a large hospital with a high prevalence (ie, among about a dozen reasons I gave that favor false-negative results).

Regarding the former concern, they say increasing the sample size wouldn't effectively increase power, but readers of this journal probably know better. Rosner's Fundamentals of Biostatistics, 3rd edition, states that power increases "as sample size increases." ${ }^{\text {"(p209) }}$ Huskins et al. ${ }^{1}$ then defend the trial's intentionally marginal power (ie, power to detect only a $40 \%$ reduction), saying active detection and isolation cost more and thus should be required to show a bigger bang for the extra bucks, but some say the most expensive measure really is one that doesn't work, and 14 studies have reported cost savings using active detection and isolation of methicillinresistant Staphylococcus aureus (MRSA) or vancomycinresistant Enterococcus (VRE). ${ }^{4}$

Regarding the latter concern, Huskins et al. ${ }^{1}$ say cluster randomization was necessary because spread occurs throughout an ICU and could confound a trial randomizing individual patients; this observation is correct, but fails to address spread throughout the hospital and entire healthcare system that could confound the single-ICU intervention. A study by da Silva et al. showed that spread extends far beyond a single unit; $80 \%$ of cases of MRSA bacteremia in 12 hospitals across 7 states from New York to Georgia were due to just 2 clones. ${ }^{5}$ For example, an unisolated, uncolonized patient in an ICU randomized to use isolation may be visited by a consultant or technician carrying equipment contaminated elsewhere in a hospital that is generally not controlling MRSA and VRE, or this patient may be transported outside the ICU for a diagnostic or therapeutic procedure and acquire MRSA or VRE as a result. The trial likely will count these as failures of ICU isolation, but it would represent confounding. Huskins et al. ${ }^{1}$ say intervening more broadly would have been 